\title{
MARTINGALE MEASURES IN THE MARKET WITH RESTRICTED INFORMATION
}

\author{
YANG JIANQI, YAN HAIFENG, AND LIU LIMIN
}

Received 7 May 2005; Accepted 31 January 2006

This paper considers the problem of the market with restricted information. By constructing a restricted information market model, the explicit relation of arbitrage and the minimal martingale measure between two different information markets are discussed. Also a link among all equivalent martingale measures under restricted information market is given .

Copyright (c) 2006 Yang Jianqi et al. This is an open access article distributed under the Creative Commons Attribution License, which permits unrestricted use, distribution, and reproduction in any medium, provided the original work is properly cited.

\section{Introduction}

The formula of Black and Scholes for the valuation of options has led to the great development of mathematical finance. Mathematical finance is attracting more and more attention of researchers. Some useful work has been done, but the majority of discussions are based on perfect markets. A perfect market includes the following conditions: (1) many buyers; (2) many sellers; (3) individual trades do not affect the market; (4) the units of goods sold by different sellers are the same; (5) there is perfect information, that is, all buyers and sellers have complete information on the price being asked and offered in other parts of the market; (6) there is perfect freedom of entry to and exit from the market. Real financial markets are imperfect markets. In fact there are some investors different to general investors in the financial market. Because of their conditions, for example, they live in the country, the investors cannot know all market information such as some invest policies, construction plans, and so on, which are known by general investors. They might only know price information of risky assets. These make the investor's information incomplete. It is well known that hedging market risk and capturing arbitrage opportunity are closed to market information. So it conforms to financial application to discuss financial markets under different information. There are several recent papers dealing with restricted information in finance. Schweizer [15] presents risk-minimizing hedging 
strategies of contingent claims under restricted information, Pham [12] researches the problem of mean-variance hedging for partially observed drift processes, and Frey and Runggaldier [5] focus on the computation of the optimal hedging strategies when asset price processes are observed at discrete random times. The utility maximization problem when only stock prices are observed was studied by Lakner [9]. Initiated by Cox and Ross [1] and Harrison and Kreps [8], the "martingale method" of pricing derivative is one of two approaches to the pricing of derivative securities. This approach consists of writing the value of the security as the expected value of the discounted payoff under a martingale measure. If the market is incomplete, then there are many equivalent martingale measures. It may be reasonable to suppose that there should be a special martingale measure which determines the prices of contingent claims. As the candidates of such measures, several martingale measures are proposed: minimal martingale measure (Föllmer and Schweizer [4]), variance-optimal martingale measure (Schweizer [17] or Delbaen and Schachermayer [3]), canonical martingale measure (Miyahara [10]), and so forth. The examples which are given by Schachermayer [14] are useful for the understanding and the investigation of the relations among the measures above. The importance of minimal martingale measure is described in Miyahara [10], and so forth. Recently, it is mentioned that minimal martingale measure is related to the exponential utility function and to the fair prices of options (see Davis [2] and Frittelli [6]). Different from those above, the paper focuses on the relation of market completeness, arbitrage, and minimal martingale measure between markets with different information, which is important to hedging contingent claims. To our knowledge, the relation has not been discussed. In the paper, the explicit relation of arbitrage and the minimal martingale measure between two different information markets are discussed by constructing restricted information markets. Also the relation of equivalent martingale measures is given under restricted information markets.

\section{Market with restricted information}

Assume that $\left(\Omega, F, \mathscr{F}_{F}, P\right)$ is a probability space with filtration. The filtration $\mathscr{F}_{\mathscr{F}}=\left(\mathscr{F}_{t}\right)_{0 \leq t \leq T}$ satisfies the following assumptions: (1) $\mathscr{F}_{\mathscr{f}}$ is right continuous, that is, $\mathscr{F}_{t}=\bigcap_{s>t} \mathscr{F}_{s} ;$ (2) $\mathscr{F}_{0}$ contains all $P$-null sets in $F$. On $(\Omega, F, \mathscr{F}, P)$, define a financial market as follows: assume that $S$ is a local bounded $d$-dimensional semimartingale. With $S$ we denote the movement of $d$ risky assets. Also assume that there is a riskless asset denoted by $B$. For simplicity we assume $B \equiv 1$ (i.e., $S$ is the discounted asset price). Assume that market participants's investment behaviors are based on their valid market information. We denote by $\mathscr{F}_{t}$ the valid market information that general investors know up to $t$. Assume in the above market, besides general information investors, that there are another investors who know less market information than general investors. We call them restricted information investors or incomplete information investors. More explicitly, we assume that the restricted information investors only acquire market information denoted by minor $\sigma$-filtration $G$ rather than $\mathscr{F}$, where

$$
\mathscr{G}=\left(\mathscr{G}_{t}\right)_{0 \leq t \leq T}, \quad \mathscr{F}_{t}^{S} \subset \mathscr{G}_{t} \subset \mathscr{F}_{t} .
$$


$\mathscr{F}^{S}=\left(\mathscr{F}_{t}^{S}\right)_{0 \leq t \leq T}$ denotes the $\sigma$-filtration generated by asset price processes $S$. The market for restricted information investors, that is, the market with information set $\mathscr{G}$, is called restricted information market.

Remark 2.1. Obviously, the assumption for $\mathscr{G}$ is reasonable. On the one hand, there are some investors who cannot achieve all the market information $\mathscr{F}$ actually and only know the restricted information $\mathscr{G}_{t} \subset \mathscr{F}_{t}$. On the other hand, if the investor did not know the past price information, he would not throw his money. So the assumption $\mathscr{F}_{t}^{S} \subset \mathscr{G}_{t}$ is also reasonable.

\section{Main results and proofs}

It is well known that market information set is an element of finance markets. Finance markets vary with the market information set $\mathscr{F}$. Obviously, the problem that market information has what influence on the market completeness and arbitrage is worth studying. In this section we will discuss it.

For $H \in\{\mathscr{F}, \mathscr{G}\}$, we recall from Grorud and Pontier [7] and Pham [12] some definitions and notations.

Definition 3.1. A probability measure $Q$ is called $H$-equivalent martingale (local martingale) measure for $S$, if $S$ is an $(H, Q)$ martingale (local martingale), and $d Q / d P \in$ $L^{2}\left(P, H_{T}\right)$.

Define

$$
\begin{aligned}
M_{e}^{2}(P, H) & =\left\{Q \sim P, \frac{d Q}{d P} \in L^{2}\left(P, H_{T}\right), S \text { is an }(H, Q) \text { martingale }\right\}, \\
M_{\text {loc }}^{2}(P, H) & =\left\{Q \sim P, \frac{d Q}{d P} \in L^{2}\left(P, H_{T}\right), S \text { is an }(H, Q) \text { local martingale }\right\}
\end{aligned}
$$

to be the equivalent martingale and the local martingale measure sets for $S$, respectively.

Definition 3.2. Let $R \in M_{\mathrm{loc}}^{2}\left(P, \mathscr{F}_{\mathrm{F}}\right)$. If for every $\mathbf{H} \in L^{2}\left(\Omega, \mathscr{F}_{T}, P\right)$, there exists an $\mathscr{F}_{0}-$ random variable $a$ and a portfolio $\mathscr{F}$-predictable $\vartheta, \vartheta \in L^{2}(\Omega \times[0, T], d R \times d[S, S])$, such that

$$
\left(C_{1}\right): \mathbf{H}=a+\sum_{i=1}^{d} \int_{0}^{T} \vartheta^{i} d S^{i},
$$

then the market is complete for general information investors.

Also, we can define market completeness for the restricted information market.

Definition 3.3. Let $R \in M_{e}^{2}(P, \mathscr{G})$. If for every $\mathbf{H} \in L^{2}\left(\Omega, \mathscr{G}_{T}, P\right)$, there exists a $\mathscr{G}_{0}$-random variable $a$ and a portfolio $\mathscr{G}$-predictable $\vartheta, \vartheta \in L^{2}(\Omega \times[0, T], d R \times d[S, S])$, such that

$$
\left(C_{2}\right): \mathbf{H}=a+\sum_{i=1}^{d} \int_{0}^{T} \vartheta^{i} d S^{i},
$$

then the market is complete for restricted information investors. 
4 Martingale measures in the market with restricted information

Remark 3.4. By the capital asset pricing basic theorem, we know that (1) if $H_{0}$ is trivial and $M_{\mathrm{loc}}^{2}(P, H)$ is singleton, the associated market is complete; (2) if $M_{\mathrm{loc}}^{2}(P, H)$ is nonempty, there is no arbitrage in the associated market; the converse result is false. But that $M_{\mathrm{loc}}^{2}(P, H)$ is nonempty is equivalent to a weaker property: the "no free lunch with vanish risk" (NFLVR in short).

The theorem below gives the relation of arbitrage between the markets with different information.

Theorem 3.5. If $M_{e}^{2}(P, \mathscr{F}) \neq \varnothing$, then $M_{e}^{2}(P, \mathscr{G}) \neq \varnothing$.

Proof. Since $M_{e}^{2}(P, \mathscr{F}) \neq \varnothing$, let $Q \in M_{e}^{2}(P, \mathscr{F})$, then we know by definition $d Q / d P \in$ $L^{2}\left(P, \mathscr{F}_{T}\right), S$ is $(\mathscr{F}, Q)$ martingale. Let $Z_{T}=d Q / d P, Z_{t}=E\left(Z_{T} / \mathscr{F}_{t}\right)$, then $Z=\left(Z_{t}\right)_{0 \leq t \leq T}$ is a strictly positive square integrable martingale on $\left(P, \mathscr{F}_{\mathcal{F}}\right)$.

Let $\zeta_{t}=E\left[Z_{T} / \mathscr{G}_{t}\right]$, obviously $\zeta_{t} \in \mathscr{G}_{t}$ and

$$
E\left[\frac{\zeta_{t}}{\mathscr{G}_{s}}\right]=E\left[\frac{E\left[Z_{t} / \mathscr{G}_{t}\right]}{\mathscr{G}_{s}}\right]=E\left[\frac{Z_{t}}{\mathscr{G}_{s}}\right]=E\left[\frac{E\left[Z_{t} / \mathscr{F}_{s}\right]}{\mathscr{G}_{s}}\right]=E\left[\frac{Z_{s}}{\mathscr{G}_{s}}\right]=\zeta_{s} .
$$

So $\zeta=\left(\zeta_{t}\right)_{0 \leq t \leq T}$ is $(P, \mathscr{G})$ martingale. Thus we can define a probability measure $\widetilde{Q}$ as follows:

$$
\frac{d \tilde{Q}}{d P}=\zeta_{T}
$$

Next, we prove $\widetilde{Q} \in M_{e}^{2}(P, \varphi)$.

By definition we only prove three points as follows.

(1) $\widetilde{Q} \sim P$. Since $Q \sim P$, from the definition of $\widetilde{Q}, \widetilde{Q} \sim P$ holds obviously.

(2) $d \widetilde{Q} / d P \in L^{2}(P, \mathscr{G})$. In fact, only note that $\zeta_{T}=E\left[Z_{T} / \mathscr{G}_{T}\right], \zeta_{T} \in \mathscr{G}_{T}$, then we have

$$
E\left(\frac{d \tilde{Q}}{d P}\right)^{2}=E\left[E\left(\frac{Z_{T}}{\varphi_{T}}\right)\right]^{2} \leq E\left[E\left(\frac{Z_{T}^{2}}{\varphi_{T}}\right)\right]=E Z_{T}^{2}<\infty .
$$

$d \widetilde{Q} / d P \in L^{2}(P, \mathscr{G})$ is proved.

(3) $S$ is a $(\widetilde{Q}, \mathscr{G})$ martingale. By Protter [13], if $Q \sim P, N_{t}=E\left[d Q / d P / H_{t}\right]$, then $S$ is $(Q, H)$ martingale if and only if $S N$ is $(P, H)$ martingale. Thus

$$
\begin{aligned}
E\left[\frac{S_{t} \zeta_{t}}{\mathscr{G}_{s}}\right] & =E\left[\frac{S_{t} E\left(Z_{t} / \mathscr{G}_{t}\right)}{\mathscr{G}_{s}}\right]=E\left[\frac{E\left(S_{t} Z_{t} / \mathscr{G}_{t}\right)}{\mathscr{G}_{s}}\right] \\
& =E\left[\frac{S_{t} Z_{t}}{\mathscr{G}_{s}}\right]=E\left[\frac{E\left(S_{t} Z_{t} / \mathscr{F}_{s}\right)}{\mathscr{G}_{s}}\right] \\
& =E\left[\frac{S_{s} Z_{s}}{\mathscr{G}_{s}}\right]=S_{s} E\left[\frac{Z_{s}}{\mathscr{G}_{s}}\right]=S_{s} \zeta_{s} .
\end{aligned}
$$

So $S \zeta$ is a martingale, and $S$ is a $(\widetilde{Q}, \mathscr{G})$ martingale. 
Remark 3.6. (1) Generally speaking, because $\mathscr{F}$-stopping times are not always $\mathscr{G}$-stopping times, $M_{\mathrm{loc}}^{2}(P, \mathscr{F}) \neq \varnothing$ does not imply $M_{\mathrm{loc}}^{2}(P, \mathscr{G}) \neq \varnothing$.

(2) Under the condition that $S$ is a bounded semimartingale, because $M_{e}^{2}(P, H)=$ $M_{\text {loc }}^{2}(P, H)$, the nonarbitrage market for general information investor is also nonarbitrage for restricted information investor. That is a result in accordance with the market fact that the investors with more information can easy gain more arbitrage opportunities than those with less information. The converse result is false certainly.

For further discussion, we recall from Schweizer [16] the definition of minimal martingale measure.

Let price process $S$ be a $P$ semimartingale with canonical decomposition $S_{t}=S_{0}+M_{t}+$ $A_{t}, M$ is a local martingale and $A$ is a predictable finite variation process.

Definition 3.7. Suppose that $S$ satisfies the structure condition (SC): $S=S_{0}+M+\lambda d\langle M$, $M\rangle$. Moreover, $\hat{Z}=\varepsilon\left(-\int \lambda d M\right)$ is a $P$-martingale, then call $\hat{P}$ defined by $\hat{Z}_{T}=d \hat{P} / d P$ minimal signal martingale measure for $S, H$-minimal martingale measure if in addition $\widehat{P} \in M_{e}^{2}(P, H)$.

Remark 3.8. If $H$-minimal martingale measure for $S$ exists, then it is unique.

For discussing the relation of minimal martingale measure between markets with different information, we introduce a property which is an equivalent definition of minimal martingale measure in essence. (see Pham [11] for detail)

Lemma 3.9. An equivalent martingale $\hat{P}$ is a minimal martingale measure for $S$ if and only if any square integrable martingale under $P$ and orthogonal to $M$ remains a martingale under $\hat{P}$.

THEOREM 3.10. If every $\varphi$ martingale is also an F martingale, $\hat{P}^{\mathscr{F}}$ is an $\mathscr{F}$-minimal martingale measure, $Z$ is $\hat{P}^{\mathscr{F}}$ 's density process with respect to $P$, then $\hat{P}^{G}$ defined by (3.5) is also a $G$-minimal martingale measure.

Proof. Let $S=S_{0}+M+A, K$ be a square integrable $(P, \mathscr{G})$ martingale orthogonal to $M$. Thus $K$ is also a square integrable $(P, \mathscr{F})$ martingale. Using Lemma 3.9, we only prove that $K$ is a $\left(\hat{P}^{\mathscr{G}}, \mathscr{G}\right)$ martingale.

By the property of conditional expectation and the definition of martingale, for all $t>s$,

$$
\begin{aligned}
E\left[\frac{K_{t} \zeta_{t}}{\mathscr{G}_{s}}\right] & =E\left[\frac{K_{t} E\left(Z_{t} / \mathscr{G}_{t}\right)}{\mathscr{G}_{s}}\right]=E\left[\frac{E\left(K_{t} Z_{t} / \mathscr{G}_{t}\right)}{\mathscr{G}_{s}}\right] \\
& =E\left[\frac{K_{t} Z_{t}}{\mathscr{G}_{s}}\right]=E\left[\frac{E\left(K_{t} Z_{t} / \mathscr{F}_{s}\right)}{\mathscr{G}_{s}}\right] \\
& =E\left[\frac{K_{s} Z_{s}}{\mathscr{G}_{s}}\right]=K_{s} E\left[\frac{Z_{s}}{\mathscr{G}_{s}}\right]=K_{s} \zeta_{s} .
\end{aligned}
$$

So $K \zeta$ is $P$-martingale, By Protter [13, Lemma, page 109], $K$ is $\left(\hat{P}^{\mathscr{G}}, \mathscr{G}\right)$ martingale. Theorem 3.10 follows immediately from Lemma 3.9.

Obviously, from the definitions of completeness, we know that under the condition $\mathscr{F}_{T}=\mathscr{G}_{T}$, if restricted information market is complete, then general information market 
is also complete. Generally speaking, there is not close relation of completeness between markets with different information. But there is really a link among all the equivalent martingale in $M_{\mathrm{loc}}^{2}(P, \mathscr{G})$.

THEOREM 3.11. In case of a complete market for the restricted information investor (i.e., verifying $\left(C_{2}\right)$ ) such that there exists $Q \in M_{\mathrm{loc}}^{2}(P, G)$ for which the discounted prices $S$ are $\left(Q, \mathscr{F}^{S}\right)$-martingales, then every $R \in M_{\mathrm{loc}}^{2}(P, \mathscr{G})$ is equal to $f \cdot Q$, where $f \in L^{1}\left(\mathscr{F}_{0}^{S}, Q\right)$.

Proof. For all $R \in M_{\mathrm{loc}}^{2}(P, \mathscr{G})$, let $d R / d Q=Z_{T}, Z_{t}=E_{Q}\left[Z_{T} / \mathscr{F}_{t}^{S}\right]$, so we only prove $Z_{T} \in$ $L^{1}\left(\mathscr{F}_{0}^{S}, Q\right)$. Let $Q \in M_{\text {loc }}^{2}(P, \mathscr{G})$, but $S$ is an $R$-local martingale, thus $S^{i} Z$ is a $Q$-local martingale, By Itô formula, $\left[S^{i}, Z\right]=S^{i} Z-\int S_{-}^{i} d Z-\int Z_{-} d s^{i}$. Note that $S^{i} Z$ is a $Q$-local martingale, we have get $\left[S^{i}, Z\right]=0$, thus $Z$ is orthogonal to price processes $S$. Since the market is complete for the restricted information investor, there exist a $\mathscr{G}_{0}$-random variable $a$ and a $\mathscr{G}_{0}$-predictable portfolio $\varphi \in L^{2}(\Omega \times[0, T], d R \otimes[S])$ such that $Z_{T}=a+\sum_{i=i}^{d} \int_{0}^{T} \varphi^{i} d S^{i}$.

Because $S^{i}$ is an $\left(\mathscr{F}^{S}, Q\right)$-martingale, $\varphi \cdot S$ is an $\left(\mathscr{F}^{S}, Q\right)$-martingale. Thus $Z_{t}-Z_{0}=$ $\sum_{i=i}^{d} \int_{0}^{t} \varphi^{i} d S^{i}$ is strongly orthogonal to the stable space generalized by prices, then it is orthogonal to itself; therefore $Z_{t}-Z_{0}=0$ and $Z_{T}$ is an $\mathscr{F}_{0}^{S}$-measurable random variable.

Remark 3.12. By martingale pricing method, the price of a contingent claim is its expected payoff under a special equivalent martingale measure. Because there are many equivalent martingale measures in an incomplete market, there are many nonarbitrage price for a contingent claim. Under an explicit market, we can deduce the link among many arbitrage-less prices using Theorem 3.11.

\section{Conclusion}

The paper discusses an imperfect market with restricted information. Based on constructing restricted markets and martingale theory, we strictly prove the result that nonarbitrage market for general information investors is also nonarbitrage for restricted information investors. Also the explicit relation of minimal martingale measure between two different information markets is given. Finally, a link among all equivalent martingale measures in the class of restricted local martingale measures, which is important in pricing contingent claims, is derived.

\section{Acknowledgments}

This research is supported by Henan Province Science and Technology Commission Soft Science Research Project (0313062400) and Hunan University of Science and Engineering Science Research Project. We thank the three referees for their remarks on the first draft of this paper.

\section{References}

[1] J. C. Cox and S. Ross, The valuation of options for alternative stochastic processes, Journal of Financial Economics 3 (1976), no. 1-2, 145-166.

[2] M. H. A. Davis, Option pricing in incomplete markets, Mathematics of Derivative Securities (Cambridge, 1995) (M. A. H. Dempster and S. R. Pliska, eds.), Publ. Newton Inst., vol. 15, Cambridge University Press, Cambridge, 1997, pp. 216-226. 
[3] F. Delbaen and W. Schachermayer, The variance-optimal martingale measure for continuous processes, Bernoulli 2 (1996), no. 1, 81-105.

[4] H. Föllmer and M. Schweizer, Hedging of contingent claims under incomplete information, Applied Stochastic Analysis (London, 1989) (M. H. A. Davis and R. J. Elliot, eds.), Stochastics Monogr., vol. 5, Gordon and Breach, New York, 1991, pp. 389-414.

[5] R. Frey and W. J. Runggaldier, Risk-minimizing hedging strategies under restricted information: the case of stochastic volatility models observable only at discrete random times, Mathematical Methods of Operations Research 50 (1999), no. 2, 339-350.

[6] M. Frittelli, The minimal entropy martingale measure and the valuation problem in incomplete markets, Mathematical Finance 10 (2000), no. 1, 39-52.

[7] A. Grorud and M. Pontier, Asymmetrical information and incomplete markets, International Journal of Theoretical and Applied Finance 4 (2001), no. 2, 285-302.

[8] J. M. Harrison and D. M. Kreps, Martingales and arbitrage in multiperiod securities markets, Journal of Economic Theory 20 (1979), no. 3, 381-408.

[9] P. Lakner, Optimal trading strategy for an investor: the case of partial information, Stochastic Processes and Their Applications 76 (1998), no. 1, 77-97.

[10] Y. Miyahara, Canonical martingale measures of incomplete assets markets, Probability Theory and Mathematical Statistics: Proceedings of the 7th Japan-Russia Symposium (Tokyo, 1995) (S. Watanabe, M. Fukushima, Yu. V. Prohorov, and A. N. Shiryaev, eds.), World Scientific, New Jersey, 1996, pp. 343-352.

[11] H. Pham, On quadratic hedging in continuous time, Mathematical Methods of Operations Research 51 (2000), no. 2, 315-339.

[12] _ Mean-variance hedging for partially observed drift processes, International Journal of Theoretical and Applied Finance 4 (2001), no. 2, 263-284.

[13] P. Protter, Stochastic Integration and Differential Equations, Applications of Mathematics (New York), vol. 21, Springer, Berlin, 1990.

[14] W. Schachermayer, A counterexample to several problems in the theory of asset pricing, Mathematical Finance 3 (1993), 217-229.

[15] M. Schweizer, Risk-minimizing hedging strategies under restricted information, Mathematical Finance 4 (1994), no. 4, 327-342.

[16] __ On the minimal martingale measure and the Föllmer-Schweizer decomposition, Stochastic Analysis and Applications 13 (1995), no. 5, 573-599.

[17] _ Variance-optimal hedging in discrete time, Mathematics of Operations Research 20 (1995), no. 1, 1-32.

Yang Jianqi: Department of Mathematics, Hunan University of Science and Engineering, Yongzhou, Hunan 425006, China

E-mail address: yjqyyy@yahoo.com.cn

Yan Haifeng: School of Finance and Banking, Nanjing University of Finance and Economics,

Nanjing, Jiangsu 210046, China

E-mail address: yseaf@163.com

Liu Limin: Department of Mathematics, Henan Normal University, Xinxiang, Henan 453008, China

E-mail address: liulimin1971@sohu.com 


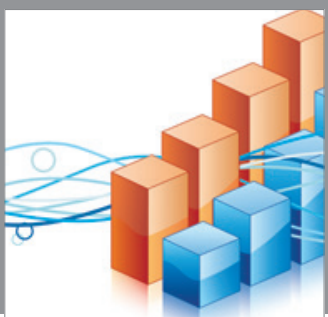

Advances in

Operations Research

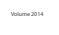

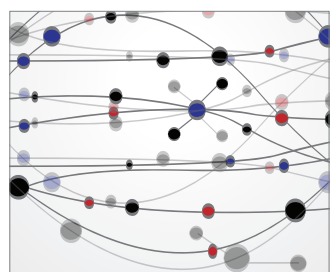

\section{The Scientific} World Journal
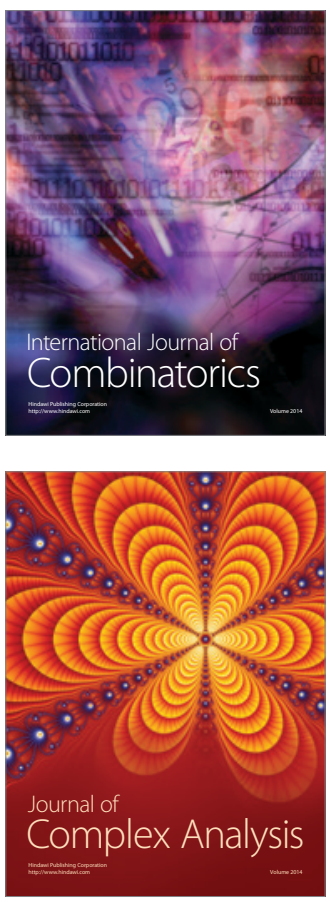

International Journal of

Mathematics and

Mathematical

Sciences
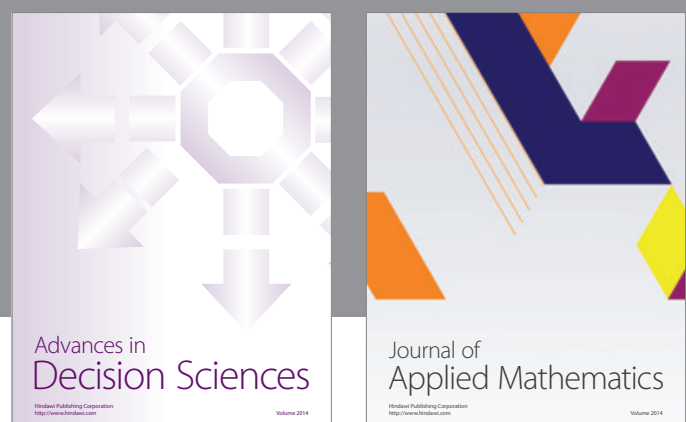

Journal of

Applied Mathematics
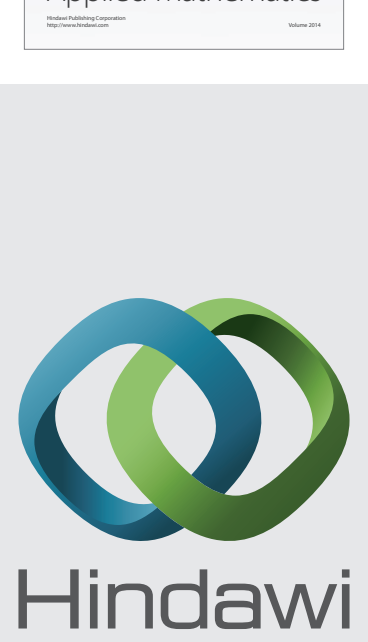

Submit your manuscripts at http://www.hindawi.com
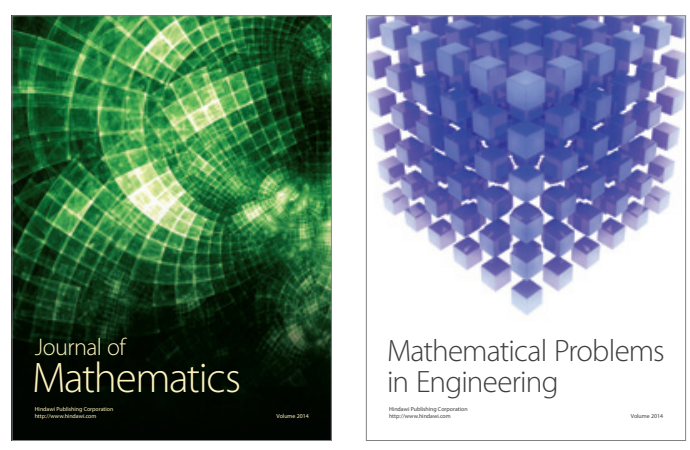

Mathematical Problems in Engineering
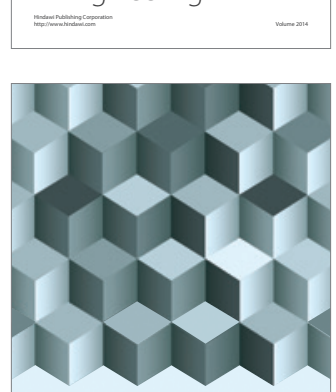

Journal of

Function Spaces
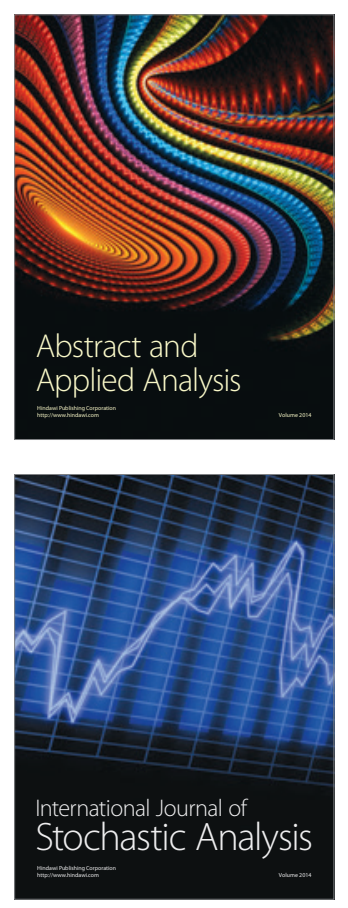

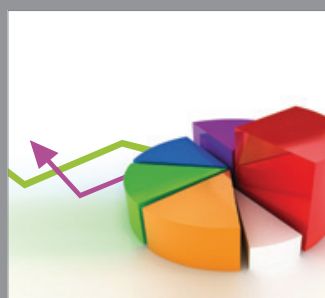

ournal of

Probability and Statistics

Promensencen
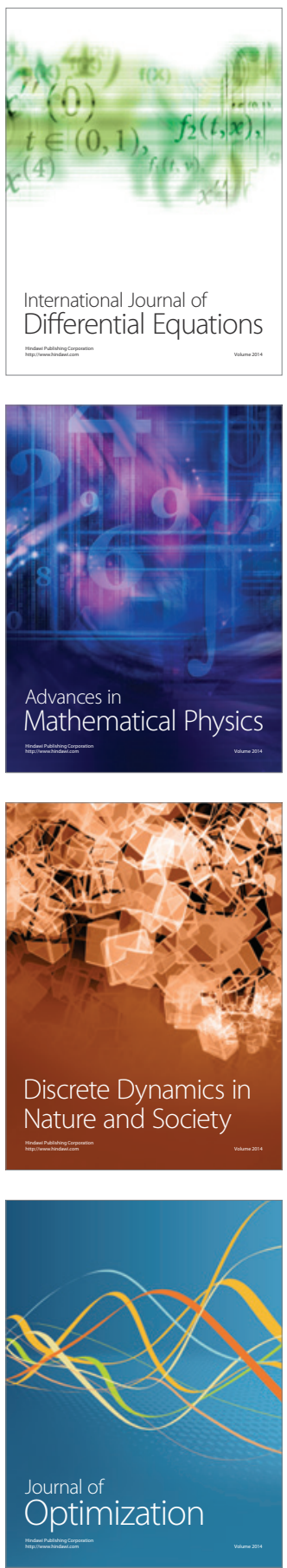